\title{
PROPIEDADES DEL SUELO EN BOSQUE Y PAJONAL; RESERVA NATURAL PUEBLO VIEJO, NARIÑO, COLOMBIA
}

\section{SOIL PROPERTIES IN FOREST AND GRASSLANDS; PUEBLO VIEJO NATURAL RESERVE, NARIÑO, COLOMBIA}

Patricia Cerón ${ }^{1}$

Hernán García²

\section{RESUMEN}

Se realizó un estudio exploratorio con el objetivo de comparar algunas propiedades químicas, físicas y biológicas de los suelos con cobertura en bosque de ladera (Bosque1), bosque en la vertiente del cauce de agua (Bosque2) y Pajonal, situados a similar altura, en la Reserva Natural Pueblo Viejo (Nariño). Se tomaron tres muestras de suelo por cada sitio, para un total de nueve muestras. Los datos, se procesaron mediante pruebas no paramétricas y Análisis de Componentes Principales. Se presentan diferencias estadísticas significativas entre Bosque2 y Pajonal, en C, N, CIC y Al, densidad aparente y densidad de Mollusca y, entre Bosque1 y Pajonal, en Mn y Cu. El análisis multivariado diferencia tres grupos correspondientes a los tres sitios.

Palabras clave: Suelos, pajonal, bosque alto andino, Andes, Nariño.

\section{SUMMARY}

An exploratory study was carried out in order to compare the chemical, physical and biological properties of vegetation-covered soils in ladder woodlands (Afforested

${ }^{1}$ Mg. Ciencias Agrarias. Docente Universidad de Nariño. Calle 23 N. 4 este 11 manzana K, casa 2, Campiña de oriente, Pasto, Nariño; patriciac1@hotmail.com

${ }^{2}$ Mg. Estadística. Docente Universidad de Nariño. Calle 13 A, \# 41.09, Villas de San Rafael, Pasto, Nariño; hgarcia@ udenar.edu.co area No.1), woodlands located in the drainage area of a water source (Afforested area No.2) and grassland, situated at the same altitude, in the 'Pueblo Viejo' Nature Reserve. Three soil samples were taken in each location, with a total of nine samples. The data were processed using non-parametric tests and Principal Component Analysis. There are significant statistical differences between Afforested area No.2 and grassland of levels of $\mathrm{C}, \mathrm{N}, \mathrm{CEC}, \mathrm{Al}$, bulk density and density of Mollusca, and between grassland and afforested area No.1 in levels of $\mathrm{Mn}$ and $\mathrm{Cu}$. The multivariate analysis considered three groups, corresponding to the three locations.

Key words: Soils, grassland, woodland of the High Andes, Andes, Nariño.

\section{INTRODUCCIÓN}

Las investigaciones sobre los suelos en los ecosistemas de alta montaña se han centrado en los páramos, siendo poco frecuentes las comparaciones de las características físicas, químicas y biológicas, entre áreas silvestres de páramo y bosque altoandino, a similar altitud.

La edafofauna, al parecer, presenta particularidades, si se trata del páramo o bosque alto aldino. Chamorro E Zuluaga (1998) encontraron que el páramo muestra un índice de riqueza en los principales taxa edáficos de 0,72, mientras que en bosque alto andino es de 0,49. Rangel-Ch. \& Sturm (1994) estimaron que el bosque presenta un mayor número total de individuos y de grupos taxonómicos en comparación con el páramo. 
En lo concerniente a las propiedades químicas, en un estudio sobre los almacenamientos de carbono y flujo de gases, se halló mayor contenido de $\mathrm{C}$ superficial en bosque frente al páramo, contrario a lo esperado, lo que fue explicado como consecuencia de las continuas quemas naturales e inducidas en el páramo. El bosque, se comportó como un sumidero neto de metano atmosférico (-33mg $\mathrm{CH}_{4} \mathrm{~m}^{2} / \mathrm{h}$ ), mientras que el páramo lo hizo como una fuente neta $\left(82 \mathrm{mg} \mathrm{CH}_{4} \mathrm{~m}^{2} / \mathrm{h}\right)$ de dicho gas (Rondón et al. 2002).

A pesar de la escasez de estudios que comparan las propiedades de los suelos entre páramo y bosque, en Ecuador se vienen adelantando investigaciones en suelos de los Andes, que coadyuvan a contextualizar los de Pueblo Viejo, debido a que el área de estudio se ubica en la zona del complejo volcánico Galeras - Chiles, entre ellos Azufral, Cerro Mayasquer, Chiles, Cumbal, Galeras, Serranía de Colimba y Túquerres, algunos con actividad volcánica histórica (menos de 500 años) y evidencia geomorfológica de actividad en el Holoceno (Robertson et al. 2002). Dado que el complejo volcánica se encuentra cerca, influye en parte de los suelos estudiados en Ecuador, lo que posibilita equiparar factores de formación del suelo, como el material parental y el tiempo (cenizas volcánicas del Holoceno).

A partir de las investigaciones mencionadas, se conoce que los suelos de ecosistemas de alta montaña de los Andes, situados en zonas volcánicas con actividad en el Holoceno, deben buena parte de sus propiedades a la meteorización de la ceniza volcánica en interacción con el clima frío y húmedo. Una fuente de variación en la distribución de los minerales es la distancia de la zona volcánica y, por tanto, de la cantidad de ceniza depositada. En los páramos de la cuenca del río Paute, se identificó una gradual disminución de las propiedades ándicas de norte a sur, que coincide con el distanciamiento de la influencia de ceniza volcánica (Buytaert et al. 2007). Los suelos más cercanos son Andisoles no alófanicos, en los cuales, el horizonte mineral superior está dominado por complejos organometálicos de $\mathrm{Al}$ y Fe y es bajo el contenido de alófana y caolín recientes, posiblemente, porque el clima húmedo y frío favorece la lixiviación de cationes básicos y el sílice liberado por la meteorización y proporciona una descomposición retardada de la materia orgánica del suelo. Con la profundidad, la alófana tiende a aumentar, lo que se atribuye a diversas causas, entre ellas, un mayor suministro de sílice, menos materia orgánica, por tanto, menor complejación y una meteorización más avanzada (Buytaert et al. 2005).

En esta dirección, varias propiedades del horizonte A, como los valores elevados de CIC, gran capacidad de retención hídrica, alta porosidad, baja densidad, porosidad fina y el carácter melánico, se relacionaron con los altos contenidos de coloides orgánicos (Poulenard et al. 2003). La acumulación de materia orgánica en el páramo, se atribuye a las condiciones de vegetación y al clima frío y húmedo a gran altura. Buytaert et al. (2007) expresan que es un efecto del clima que no depende necesariamente del aluminio del suelo, puesto que en la cuenca del río Paute registraron similar contenido de $C$ entre el norte, con mayor nivel de aluminio libre $\mathrm{y}$, el sur, con disminución, sugiriendo entonces, que la mayor precipitación en el sur puede compensar la alta complejación organómetálica en el norte. Otros autores consideran que condiciones edáficas, como el pH ácido, el dominio de complejos de humus-Al (Zehetner et al. 2003) y la predominancia de los microporos asociados con la abundancia de coloides orgánicos coadyuvan en este proceso (Poulenard et al. 2003). El agua y la aireación del suelo pueden ser propiedades a tener en cuenta, dado que se conoce que en el páramo la mayor cantidad de materia orgánica se relaciona con la elevada capacidad de almacenamiento de agua (Buytaert et al. 2007, Poulenard et al. 2003) y a que Rondón et al. (2002) encontraron que el páramo se comporta como una fuente neta de metano, indicando que prevalecen condiciones anaeróbicas en las capas superficiales del suelo, mientras que el bosque actúa como un importante sumidero neto de metano atmosférico, indicador de condiciones aeróbicas.

Los suelos derivados de ceniza volcánica también están afectados por la variación altitudinal del clima, con lo cual, las propiedades ándicas aumentan con la altitud. En las laderas del volcán Cotacachi, se detectó que por encima de $3.200 \mathrm{~m}$, la fracción coloidal es dominada por complejos de humus-Al y alófana; a esta altura, se registra una alta absorción de fósforo, aumentan los contenidos de limo, arcilla, C, CIC y disminuye arena, $\mathrm{pH}, \mathrm{P}$ disponible, suma de bases intercambiables y las tasas de escorrentía y erosión del suelo. A elevaciones intermedias (3.200 - 2.700), se marca una zona de transición en la que coexisten alófana y halloisita. Por debajo de $2.700 \mathrm{~m}$ halloisita es el principal constituyente 
coloidal y disminuye la alófana; las variables de fertilidad innata, erosión y escorrentía siguen la tendencia contraria a los suelos, por encima de los 3.200 metros. El patrón de altitud, se percibió alterado en posiciones de paisaje inestable donde las propiedades ándicas del suelo han sido modificadas por la erosión de la capa superficial del suelo. El clima fue considerado el principal factor responsable de las diferencias de altitud en el suelo, porque afectan el régimen de lixiviación y la descomposición de materia orgánica (Zehetner et al. 2003, Zehetner \& Miller, 2006).

Conceptualmente, se prevén propiedades particulares entre los suelos de Bosque altoandino y páramo; sin embargo, hay poca información experimental que evidencie las diferencias. En este contexto, se realizó un estudio exploratorio, con el objetivo de comparar algunas propiedades físicas y químicas, y la macrofauna del suelo con cobertura en pajonal, bosque en ladera y bosque en vertiente de agua, situados a similar altitud, en la Reserva Natural Pueblo Viejo.

\section{MATERIALES Y MÉTODOS}

La Reserva Natural Pueblo Viejo está localizada en el suroeste de Colombia, Departamento de Nariño, municipio de Mallama. Las coordenadas geográficas extremas son $1^{\circ} 3^{\prime} 33,7^{\prime \prime}$ y $1^{\circ} 1^{\prime} 39,8^{\prime}$ ' Norte y $77^{\circ} 48^{\prime} 3,3^{\prime \prime}$ y $77^{\circ} 46^{\prime} 17,2^{\prime \prime}$ ' Oeste. La temperatura promedio es de $10,9^{\circ} \mathrm{C}$, presentando el valor mínimo $\left(9,3^{\circ} \mathrm{C}\right)$, en el mes de agosto y el máximo $\left(12,3^{\circ} \mathrm{C}\right)$, en abril; la precipitación total anual de la zona es de $961 \mathrm{~mm}$, datos registrados por el Instituto de Hidrología, Meteorología y Estudios Ambientales (IDEAM, 2006) en la estación El Paraíso, municipio de Túquerres. Los suelos, se clasifican como Andosoles y los bosques presentan entre 15 a $20 \mathrm{~cm}$ de hojarasca y pajonal, entre 1 a $5 \mathrm{~cm}$, seguido de $50 \mathrm{~cm}$ de horizonte mineral de color negro, con presencia de raíces y de piedra pómez pequeña.

Hay tendencia a que el área occidental de la reserva el bosque altoandino ascienda a mayor altitud, mientras que a la misma altura, en las áreas central y oriental, predomina el páramo, excepto alrededor de los cauces de las quebradas, donde también se encuentra bosque. En tal medida, se establecieron tres sitios: Bosque1, que hace parte de la ladera, en el área occidental de la reserva, Pajonal, ubicado en el área central y, Bosque2, en el área oriental, en el cauce de la cuenca alta de la quebrada Honda, que presenta agua intermitentemente. Las muestras de suelo y de macrofauna, se colectaron durante agosto, entre los 3.300 y 3.350m de altitud, en pendientes entre 18 a $20^{\circ}$. De cada sitio, se tomaron tres muestras separadas, por al menos $5 \mathrm{~m}$, para un total de nueve muestras de suelo.

Bosque1 y Bosque 2 contienen arbustos y arbolitos, entre los que se encuentran Gynoxis sanctii-antonii Cuatr., Lepichinia vulcanicola Word, Zanthoxylum quinduensi (Tul), Baccharis buddlejoides H.B.K., Hesperomeles sp, Oreopanax bogotensis Cuat., Euphorbia laurifolia Lam., Morella parvifolia Benth. El Pajonal hace parte del páramo, con vegetación herbácea formadora de macollas, dominada por la familia Poaceae, especialmente del género Calamagrostis asociada a otros géneros como Rhynchospora y Castilleja. Comprende algunas especies, como Hypericum junipericum Kunth, Hypericum laricifolium Juss, Lachemilla mutisi Roth, Hypoacheris sessiliflora Kunth, Cortaderia nítida (Kung) Pilg., Monnina aestuans (LF) DC., Bidens andicola H.B.K., Rhynchospora macrochaeta L. y, Pernettya postrata (Cav). D.C.

Las muestras de suelo, se tomaron de 0 a $20 \mathrm{~cm}$ de profundidad, alrededor de los monolitos, de los que se extrajeron la macrofauna. Se homogeneizó la muestra, se trasladó al laboratorio de suelos de la Universidad de Nariño, para estimar las siguientes variables: textura (Bouyoucos), humedad higroscópica (secado a $105^{\circ} \mathrm{C}$ hasta peso constante), densidad real (picnómetro), densidad aparente (cilindro graduado) porosidad, $\mathrm{pH}$ (potenciómetro, 1:1), carbono orgánico (WalkleyBlack), fósforo (Bray II), nitrógeno total (estimación de la M.O.), calcio, magnesio, potasio, capacidad de intercambio catiónico (AcNH4O 1N pH 7), aluminio (KCl $1 \mathrm{~N})$, hierro, manganeso, cobre, zinc (DTPA) y boro (agua caliente). La macrofauna, se obtuvo de tres monolitos que consistían en la hojarasca y suelo en $25 \mathrm{~cm} \times 25 \mathrm{~cm} \times 30 \mathrm{~cm}$ de profundidad, para cada uno de los sitios seleccionados (Bosque1, Pajonal y Bosque2); fue extraída manualmente, se fijó en alcohol y se procedió a cuantificar su densidad y estimar la biomasa, como peso fresco, aplicando la corrección por pérdida de humedad (19\% para lombriz de tierra, 11\% para coleópteros, $6 \%$ para arácnidos y miriápodos, $13 \%$ para otros invertebrados), como lo sugieren Decaëns et al. (1994). 
Para identificar si se presentan diferencias estadísticas significativas en las características químicas, físicas y la macrofauna entre los tres sitios, se realizó un análisis de varianza no paramétrico de Kruskal-Wallis. En los casos donde se encontraron diferencias significativas, se llevó a cabo una prueba de comparaciones múltiples, para lo que se empleó el paquete estadístico STATISTICA. Para complementar la comparación, se efectuó una correlación de Pearson y un análisis de componentes principales (ACP) aplicando los procedimientos princomp y prinqual del paquete estadístico SAS, utilizando, como individuos, a los suelos de las diferentes localizaciones geográficas y, como variables, las siguientes propiedades: arena, humedad higroscópica, densidad real, densidad aparente, $\mathrm{pH}, \mathrm{C}, \mathrm{P}, \mathrm{Ca}, \mathrm{Mg}, \mathrm{K}, \mathrm{CIC}, \mathrm{Al}, \mathrm{Fe}, \mathrm{Cu}, \mathrm{Mn}, \mathrm{Zn}$, biomasa y densidad total de la macrofauna edáfica.

\section{RESULTADOS Y DISCUSIÓN}

En cuanto a las propiedades químicas es de anotar que los contenidos de $\mathrm{C}$, CIC, $\mathrm{N}$ y $\mathrm{Al}$ son más elevados en Pajonal, seguidos de Bosque1, con valores que no se diferencian estadísticamente, mientras que en Bosque2 hay una disminución con diferencia estadísticamente significativa de Pajonal (Tabla 1). En elementos menores, hay diferencias significativas en las variables $M n$ y Cu entre Bosque1 y Pajonal, siendo que en el segundo, la cantidad de Mn es más baja y de Cu más alta (Tabla 2).

En lo concerniente a propiedades físicas, se destaca la textura y la densidad aparente. Respecto a la textura de los suelos estudiados, se define como Arenosa Franca en Bosque1 y Pajonal y, Arenosa, en Bosque2; el promedio

Tabla 1. Propiedades químicas en los suelos estudiados.

\begin{tabular}{|c|c|c|c|c|c|c|c|c|c|}
\hline Suelos & pH & CO & N total & P & CIC & Al & Ca & Mg & K \\
\hline & H2O & \multicolumn{2}{|c|}{$\%$} & ppm & \multicolumn{5}{|c|}{ meq $100 g^{1}$} \\
\hline Bosque1 & $5,3^{\mathrm{a}}$ & $7 \mathrm{ab}$ & $0,48 \mathrm{ab}$ & $11,1^{\mathrm{a}}$ & $29,1 \mathrm{ab}$ & $0,9 \mathrm{ab}$ & $3,93^{\mathrm{a}}$ & $0,63^{\mathrm{a}}$ & $0,37^{\mathrm{a}}$ \\
\hline Pajonal & $4,8^{\mathrm{a}}$ & $12^{\mathrm{a}}$ & $0,68^{\mathrm{a}}$ & $11,8^{\mathrm{a}}$ & $46,2^{\mathrm{a}}$ & $2,0^{\mathrm{a}}$ & $2,73^{\mathrm{a}}$ & $0,33^{\mathrm{a}}$ & $0,38^{\mathrm{a}}$ \\
\hline Bosque2 & $5,2^{\mathrm{a}}$ & $3 \mathrm{~b}$ & $0,25 \mathrm{~b}$ & $34,2^{\mathrm{a}}$ & $11,1 \mathrm{~b}$ & $0,3 \mathrm{~b}$ & $2,07^{\mathrm{a}}$ & $0,27^{\mathrm{a}}$ & $0,09^{\mathrm{a}}$ \\
\hline K-W & 0,047 & 0,027 & 0,027 & 0,051 & 0,027 & 0,027 & 0,065 & 0,051 & 0,063 \\
\hline
\end{tabular}

K-W: Anova Kruskal-Wallis. En letras iguales no hay diferencia significativa en la comparación múltiple $(\alpha \leq 0,05)$.

Tabla 2. Elementos menores.

\begin{tabular}{|l|c|c|c|c|c|}
\hline \multicolumn{1}{|c|}{ Suelos } & $\mathrm{Fe}$ & $\mathrm{Mn}$ & $\mathrm{Cu}$ & $\mathrm{Zn}$ & $\mathrm{B}$ \\
\hline & \multicolumn{5}{|c|}{$\mathrm{ppm}$} \\
\hline Bosque1 & $30,67^{\mathrm{a}}$ & $4,37^{\mathrm{a}}$ & $0,19 \mathrm{~b}$ & $0,38^{\mathrm{a}}$ & $0,08^{\mathrm{a}}$ \\
\hline Pajonal & $34,67^{\mathrm{a}}$ & $1,17 \mathrm{~b}$ & $0,33^{\mathrm{a}}$ & $0,47^{\mathrm{a}}$ & $0,13^{\mathrm{a}}$ \\
\hline Bosque2 & $19,33^{\mathrm{a}}$ & $1,77 \mathrm{ab}$ & $0,23 \mathrm{ab}$ & $0,22^{\mathrm{a}}$ & $0,13^{\mathrm{a}}$ \\
\hline K-W & 0,046 & 0,035 & 0,026 & 0,051 & 0,298 \\
\hline
\end{tabular}

K-W: Anova Kruskal-Wallis. En letras iguales no hay diferencia significativa en la comparación múltiple $(\alpha \leq 0,05)$.

de densidad aparente es más alto en Bosque2 y más bajo en Pajonal, con diferencias estadísticas significativas entre los dos sitios (Tabla 3).

La macrofauna edáfica pertenece a los Phylla Annelida, Arthropoda y Mollusca; la primera está constituida por la clase Oligochaeta; la Mollusca por la clase Gastropoda y, finalmente la Arthropoda por Chilopoda, Diplopoda, Arachnida, Malacostraca e Insecta. La densidad total de la macrofauna y de los artrópodos es más alta en Bosque2, intermedia en Bosque1 y más baja Pajonal; la densidad de anélidos fue mayor en Pajonal, seguida 
Tabla 3. Propiedades físicas de los suelos estudiados.

\begin{tabular}{|c|c|c|c|c|c|c|}
\hline Suelos & Textura & Arena & $\begin{array}{c}\text { Humedad } \\
\text { Higroscópica }\end{array}$ & Porosidad & Densidad real & $\begin{array}{c}\text { Densidad } \\
\text { Aparente }\end{array}$ \\
\hline & \multicolumn{7}{|c|}{$\%$} & \multicolumn{2}{|c|}{$\mathrm{g} / \mathrm{cc}$} \\
\hline Bosque1 & AF & $73^{\mathrm{a}}$ & $0,96^{\mathrm{a}}$ & $66^{\mathrm{a}}$ & $2,4^{\mathrm{a}}$ & $0,8 \mathrm{ab}$ \\
\hline Pajonal & AF & $75^{\mathrm{a}}$ & $0,93^{\mathrm{a}}$ & $69^{\mathrm{a}}$ & $2,2^{\mathrm{a}}$ & $0,7 \mathrm{~b}$ \\
\hline Bosque2 & A & $89^{\mathrm{a}}$ & $1,00^{\mathrm{a}}$ & $55^{\mathrm{a}}$ & $2,5^{\mathrm{a}}$ & $1,1^{\mathrm{a}}$ \\
\hline K-W & & 0,063 & 0,698 & 0,050 & 0,064 & 0,020 \\
\hline
\end{tabular}

K-W: Anova Kruskal-Wallis. En letras iguales no hay diferencia significativa en la comparación múltiple $(a \leq 0,05)$.

de Bosque2 y, en menor medida, en Bosque1. Los moluscos, se encontraron principalmente en Bosque2 y no se registraron en Pajonal, con diferencias estadísticamente significativas entre estos dos sitios. La biomasa está siendo aportada por artrópodos y por anélidos y teniendo en cuenta el sitio, es más alta en Pajonal, con el $53 \%$ de la biomasa total, donde Annelida constituye el $68 \%$ del peso, mientras que es similar en los bosques. En Bosque1 contribuyen, los artrópodos; en Pajonal, los anélidos y los artrópodos y en Bosque2, los artrópodos y los moluscos. No se presentaron diferencias estadísticamente significativas (Tabla 4).
En el análisis de componentes principales, se retuvieron los dos primeros factores que, en conjunto, explican el $74 \%$ de la variabilidad total de los datos. En el primer componente, con el $50 \%$, las variables que más aportan, en forma positiva, son CIC $(0,974)$, C $(0,963)$, Al $(0,935)$, Fe $(0,857)$ y, en forma negativa, densidad aparente $(-0,954), P(-0,868)$ y densidad real $(0,840)$, que corresponde con Pajonal; en el segundo componente, que explica $24 \%$, contribuyen $M n(0,969), M g(0,879)$, $\mathrm{pH}(0,801)$ y Ca $(0,797)$, asociadas a Bosque2. La figura 1 permite hacer un estudio conjunto de los individuos o sitios geográficos y las variables. Los sitios geográficos,

Tabla 4. Promedio de biomasa y densidad de macrofauna.

\begin{tabular}{|c|c|c|c|c|c|c|c|c|}
\hline \multirow{2}{*}{$\begin{array}{l}\text { Biomasa } \\
\text { (g.p.f m²) }\end{array}$} & \multicolumn{2}{|c|}{ Arthropoda } & \multicolumn{2}{|c|}{ Annelida } & \multicolumn{2}{|c|}{ Mollusca } & \multicolumn{2}{|c|}{ Total } \\
\hline & No. & $\%$ & No. & $\%$ & No. & $\%$ & No. & $\%$ \\
\hline Bosque1 & $7,0^{a}$ & 90,1 & $0,7^{a}$ & 9,6 & $0,02^{\mathrm{a}}$ & 0,3 & $8^{a}$ & 23 \\
\hline Pajonal & $5,7^{a}$ & 31,7 & $12,2^{\mathrm{a}}$ & 68,3 & $0,00^{\mathrm{a}}$ & 0,0 & $18^{a}$ & 53 \\
\hline Bosque2 & $5,9^{a}$ & 73,6 & $0,5^{\mathrm{a}}$ & 6,3 & $1,62^{\mathrm{a}}$ & 20,1 & $8^{a}$ & 24 \\
\hline Promedio & 18,6 & 55,2 & & 40,0 & 1,64 & 4,9 & 34 & 100 \\
\hline$a \leq 0.05^{* *}$ & 0,733 & & 0,276 & & 0,076 & & 0.733 & \\
\hline \multicolumn{9}{|c|}{ Densidad* (ind $\mathrm{m}^{2}$ ) } \\
\hline Bosque1 & $491^{a}$ & 94 & $5^{a}$ & 1 & $27 a b$ & 5 & $523^{a}$ & 25 \\
\hline Pajonal & $144^{a}$ & 54 & $123^{\mathrm{a}}$ & 46 & $0 \mathrm{~b}$ & 0 & $267^{a}$ & 13 \\
\hline Bosque2 & $720^{a}$ & 56 & $64^{a}$ & 5 & $491^{a}$ & 38 & $1275^{a}$ & 62 \\
\hline Promedio & 1355 & 66 & 192 & 9 & 517 & 25 & 2064 & 100 \\
\hline K-W & 0,059 & & 0,101 & & 0,031 & & 0,059 & \\
\hline
\end{tabular}

K-W: Anova Kruskal-Wallis. En letras iguales no hay diferencia significativa en la comparación múltiple ( $\alpha \leq 0.05)$. 


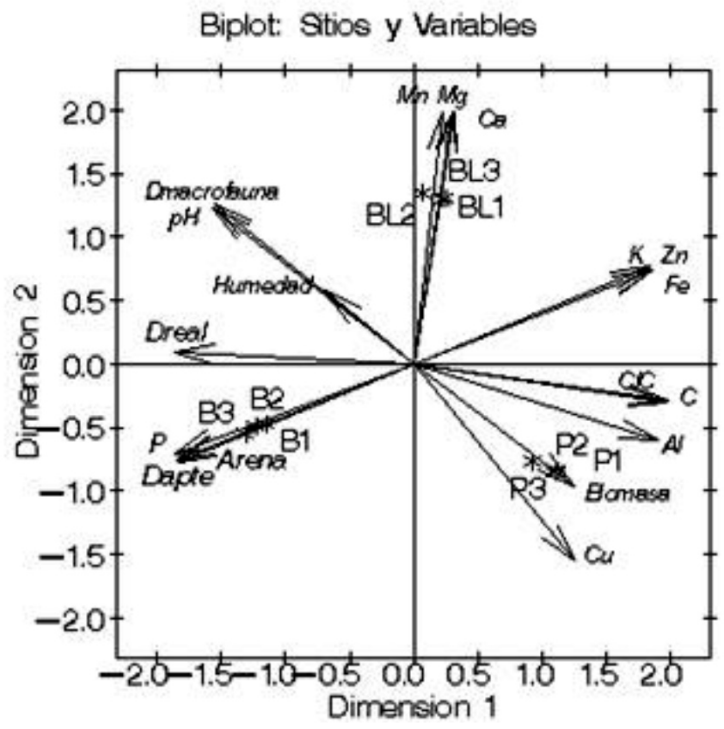

P: pajonal, BL: Bosque en ladera, B: Bosque en vertiente de fuente de agua.

Figura 1. Análisis de componentes principales.

se analizan en términos de similitud (distancias) y las variables o propiedades de los sitios geográficos, en términos de correlaciones.

En la figura, se puede observar que en cuanto a sitios, se diferencian claramente tres grupos: Pajonal (P1, P2 y P3), Bosque1 (BL1, BL2 y BL3) y Bosque2 (B1, B2 y B3). Se aprecia que Pajonal tiende a mayores niveles de biomasa total de macrofauna, C, CIC, Al y Cu y menores de $\mathrm{pH}$ y densidad total de macrofofauna; Bosque1, se asocia con contenidos más altos de $\mathrm{Ca}, \mathrm{Mg}$ y $\mathrm{Mn}$ y en Bosque2, se incrementa arena, $\mathrm{P}$ y densidad aparente y disminuye K, Fe y Zn.

Se define entonces, que los tres sitios evidencian características particulares en los suelos. Entre las variables que más contribuyen a la diferencia, se resaltar el $\mathrm{pH}$, que es muy fuertemente ácido en Pajonal y fuertemente ácido en los bosques; los contenidos de C, $\mathrm{N}$, CIC y Al son más elevados en Pajonal, intermedios en Bosque1 y más bajos en Bosque2. Esto corresponde a una tendencia inversa a la mostrada por la densidad total de la macrofauna y de los artrópodos, ya que decrecen en el orden: Bosque2, Bosque1, Pajonal. Por otra parte, la densidad aparente más baja, se evidencia en Pajonal y la más alta en Bosque2; la biomasa de la macrofauna se asocia a Pajonal.
En Pajonal, el pH $\left(\mathrm{H}_{2} \mathrm{O}\right)$ es menor de cinco, condición en la que se inhibe la formación de alófana (Shoji et al. 1982) y en consecuencia, se estarían favoreciendo los complejos de humus-Al. El dominio de estos complejos en el horizonte A se registra en los páramos de Ecuador con influencia de cenizas volcánicas del Holoceno (Buytaert et al. 2007; Buytaert et al. 2005, Poulenard et al. 2003, Zehetner et al. 2003). Específicamente, en El Ángel, con influencia de los Volcanes Cuicocha, Soche, Azufral y Galeras, los dos últimos en el sur de Colombia, se encontraron suelos con altos niveles de coloides orgánicos, que presentaban un efecto anti-alófana, es decir, que cuando más contenido de carbón, más bajo era el de alófana. En este orden de ideas, es probable que los contenidos más altos de $\mathrm{Al}, \mathrm{C}, \mathrm{CIC}$ y $\mathrm{N}$ estén asociados con el $\mathrm{pH}$ más ácido y con la formación de complejos de humus-Al, que, en otras palabras, pueden indicar un mayor contenido de materia orgánica frente a los bosques, porque además de tener valores más altos, dichas variables se correlacionan entre sí; por ejemplo, C, presenta correlación positiva con $\mathrm{CIC}(\mathrm{r}: 0,96 \mathrm{p}: 0,00)$ y $\mathrm{Al}(\mathrm{r}: 0,95 \mathrm{p}: 0,00) \mathrm{y}$, en forma negativa, con $\mathrm{pH}(\mathrm{r}:-0,71$ $\mathrm{p}: 0,03)$, densidad aparente (r: -0,90 p: 0.00) y densidad real (R: -0,88 p: 0,00). A la vez, el incremento de materia orgánica explica la densidad más baja en este sitio y la correlación negativa de la densidad, tanto real como aparente, con C, CIC, $\mathrm{N}$ y Al. Igualmente, en páramos 
del Ecuador, se encontró la correlación inversa entre C y densidad aparente (Buytaert et al. 2007).

Si bien no se presentan diferencias estadísticas en Pajonal frente a los bosques, prevalece la biomasa y disminuye la densidad de la macrofauna. La biomasa está siendo aportada, especialmente, por la lombriz de tierra, macroinvertebrado que parece estar adaptado a la estructura herbácea de la vegetación, pues Coral E Bonilla (2002), a una altitud entre 2.700 y 3.000m, cuantificaron una mayor densidad de lombriz de tierra en pastizal, (4.560ind $/ \mathrm{m}^{2}$ ) que aportó una biomasa de $67,1 \mathrm{~g} / \mathrm{m}^{2}$, en comparación a una selva secundaria, con $1.024 \mathrm{ind} / \mathrm{m}^{2}$ y biomasa de $18,2 \mathrm{~g} / \mathrm{m}^{2}$. La menor densidad de macrofauna en páramo frente al bosque fue explicada por Rangel-Ch. E Sturm (1994), como consecuencia de la ausencia de hojarasca y con clima más extremo en el primero, a lo que también habría que agregar las propiedades edáficas, pues como se registró en Pajonal, existen mayores contenidos de $\mathrm{C}, \mathrm{N}, \mathrm{CIC}, \mathrm{Al}$ y menor densidad aparente.

Bosque1 posee un pH mayor de cinco, condición en la que se forma alófana (Shoji et al. 1982), en tal caso y por la altitud, se puede estar presentando una coexistencia de complejos de humus-Al con alófana (Zehetner et al. 2003; Buytaert et al. 2005). Bosque2, además de la acentuada disminución de $\mathrm{C}, \mathrm{N}, \mathrm{CIC}$ y $\mathrm{Al}$, registra una mayor densidad aparente y densidad de moluscos, con diferencia estadística significativa de Pajonal y, el ACP, asocia este sitio con el incremento de arena, densidad aparente y fósforo, variables que correlacionan positivamente entre sí y negativamente con $\mathrm{C}, \mathrm{N}$ y $\mathrm{CIC}$. Es posible que las particularidades de Bosque2 estén marcadas por su posición en la vertiente del cauce de agua, de ahí que una mejor circulación y disponibilidad de agua, así como un microclima protegido de los vientos, puede coadyuvar a un mayor grado de descomposición de la materia orgánica, por lo cual, las variables asociadas aparecen con niveles más bajos. A su vez, la ubicación en la vertiente de la quebrada, explica el porcentaje más alto de arena, que contribuye al aumento de densidad. El contenido más alto de fósforo, igualmente, se puede explicar, en relación con el menor contenido de materia orgánica, pues correlaciona, en forma positiva, con densidad aparente (r: 0,96 p: 0,00) y negativa, con $C(r:-0,76$ p: 0,02), CIC (r: -0,79 p: 0,01), N (r: -0,82 p: 0,01) y Fe (r: -0,93, p: 0,00). Zehetner et al. (2003) y Zehetner $\mathcal{E}$
Miller (2006) asociaron el contenido de C y los grupos activos $\mathrm{OH}-\mathrm{Al}$ con la retención del fósforo; cuando el horizonte A registró $7,5 \%$ de $\mathrm{C}$, la fijación de $\mathrm{P}$ fue $88 \%$, pero con $3,4 \%$ de C, el P inmovilizado fue del $61 \%$. De igual forma, encontraron que el $\mathrm{P}$ disponible tenía valores de intermedio a bajo, en suelos por encima de $3.200 \mathrm{~m}$, donde aumenta la materia orgánica, mientras que el $\mathrm{P}$ se incrementaba en suelos por debajo de $2.700 \mathrm{~m}$, donde decrece la materia orgánica.

En otras palabras, esta exploración inicial conlleva a inferir que las propiedades edáficas de los tres sitios presentan particularidades en buena parte, debidas a diferencias en la dinámica de la materia orgánica, con incidencias en la macrofauna. En un lado, Pajonal con contenidos más altos de $\mathrm{C}, \mathrm{N}, \mathrm{CIC}$ y Al, y, más bajos de densidad aparente, tendería a acumular materia orgánica, relacionada con el pH más ácido y formación de complejos de Al-humus, condición en la que si bien disminuye la densidad de macrofauna, se posibilita un incremento de su biomasa. En Bosque1 habría un nivel intermedio de materia orgánica asociada a un $\mathrm{pH}$ mayor de cinco, donde pueden coexistir complejos de Al-humus con alófana. En el otro extremo, en Bosque2, habría mayor descomposición de la materia orgánica, con aumento en la densidad del total de macrofauna, dada su ubicación en la vertiente de la quebrada.

\section{CONCLUSIÓN}

Se sugiere que bosque de ladera, bosque en vertiente de fuente de agua y Pajonal, ubicados a similar altitud, presentan configuraciones edáficas particulares en los primeros $20 \mathrm{~cm}$ de profundidad. Las principales diferencias, se evidencian entre pajonal y bosque en la vertiente de la fuente de agua; el primero, con contenidos más altos en $\mathrm{C}, \mathrm{N}$, CIC y Al y más bajos en densidad aparente, y densidad de gasterópodos, con diferencias estadísticas significativas. Pajonal muestra mayor biomasa de la macrofauna, mientras que el bosque registra más densidad de macrofauna.

\section{AGRADECIMIENTOS}

A la Vicerrectoría de Investigaciones de la Universidad de Nariño, por el apoyo al proyecto "Caracterización físico biótica y antrópica de la Reserva Natural Pueblo Viejo"; a Benhur Cerón y Luis Navas, compañeros de 
investigación; a Elkin Noguera y Silvia Montenegro, estudiantes investigadores, a Fernando Fernández, de Ciencias Naturales, Universidad Nacional, Sede Bogotá.

\section{BIBLIOGRAFIA}

BUYTAERT, W.; SEVINKC, J.; De LEEUWC, B.; J. DECKERS, J. 2005. Clay mineralogy of the soils in the south Ecuadorian páramo. Geoderma 127:114-129.

BUYTAERT, W.; DECKERS, J.; WYSEURE G. 2007. Regional variability of volcanic ash soils in south Ecuador: The relation with parent material, climate and land use. Catena 70:143-154.

CHAMORRO, C.; ZULUAGA, D. 1998. Comunidades bioedáficas de tres regiones naturales de Colombia. Suelos Ecuatoriales 28:269-272.

CORAL, D.; BONILLA, C. 2002. Impacto de las prácticas agrícolas sobre la macrofauna del suelo en la cuenca alta del Lago Guamués, Pasto, Colombia. Suelos Ecuatoriales 32:157-160.

DECAËNS, T.; LAVELLE, P.; JIMÉNEZ, J.; ESCOBAR, G.; RIPPSTEIN G. 1994. Impact of land management on soil macrofauna in the Oriental Llanos of Colombia 1994. Eur. J. Soil Biol. 30(4):157-168.

POULENARD, J.; PODWOJEWSKI, P.; HERBILLON A. 2003. Characteristics of non-allophanic Andisols with hydric properties from the Ecuadorian páramos. Geoderma 117:267-281.
RANGEL-CH, O.; STURM, H. 1994. Consideraciones sobre la vegetación, la productividad primaria neta y la artropofauna asociada en regiones paramunas de la cordillera oriental. En: Mora E Sturm, eds. Estudios ecológicos del páramo y del bosque altoandino, cordillera oriental de Colombia, Tomo 1. Guadalupe, Bogotá. 47-70.

ROBERTSON, K.; FLÓREZ, A.; CEBALLOS, J.L. 2002. Geomorfología volcánica, actividad reciente y clasificación en Colombia. Cuadernos de Geografía. 11(2):37-60.

RONDÓN, M.; AMÉZQUITA, E.; DÍAZ, E.; CHÁVEZ, L.; PAZ, L.; CHÁVEZ J. 2002. Efecto de cambios en el uso del suelo sobre los almacenamientos de carbono y flujos de gases de efecto invernadero en áreas del Páramo de las Ánimas, Cauca, Colombia. En: Congreso Mundial de páramos, tomo 1. IDEAM (Bogotá). p.154-159.

SHOJI, S.; FUJIWARA, Y.; YAMADA, I.; SAIGUSA, M. 1982. Chemistry and clay mineralogy of Ando soils, Brown Forest soils, and Podzolic soils formed from recent Towada ashes, northeastern Japan. Soil Sci. 133:69-86.

ZEHETNER, F.; MILLER, W.P.; WEST, L.T. 2003, Pedogenesis of Volcanic Ash Soils in Andean Ecuador. Soil Science Soc. Am. 67:1797-1809

ZEHETNER, F.; MILLER, W.P. 2006. Soil variations along a climatic gradient in an Andean agro-ecosystem. Geoderma 137:126-134

Recibido: Junio 19 de 2008

Aceptado: Febrero 20 de 2009 